\title{
$\left(\mathrm{C}_{5} \mathrm{H}_{5} \mathrm{NO}\right)_{2} \mathrm{CuCl}_{2}$ 中 $\mathrm{Cu}^{2+}$ 零场分裂的研究*
}

\author{
李兆民孙威立 \\ (四川师范大学物理系, 成都 610066)
}

\section{关键词 $\left(\mathrm{C}_{5} \mathrm{H}_{5} \mathrm{NO}\right)_{2} \mathrm{CuCl}_{2}$ 电子能级 零场分裂 $D, E$}

过渡金属族 $d^{9}\left(d^{1}\right)$ 电子组态的离子在任何对称晶场中, 零场分裂轴对称分量 $D$ 和斜方分 量 $E$ 理论上为零. 例如, 文献 $[1 \sim 7]$ 研究了络离子中 $\mathrm{Cu}^{2+}\left(d^{9}\right)$ 离子的光学、磁学和电子顺磁 共振 (EPR) 性质, 均无 $D$ 和 $E$ 出现. 然而, Kokoszka 等 ${ }^{[8]}$ 的 EPR (Electron paramagnetic resonance) 实验发现晶体 $\left(\mathrm{C}_{5} \mathrm{H}_{5} \mathrm{NO}\right)_{2} \mathrm{CuCl}_{2}$ 中 $\mathrm{Cu}^{2+}$ 离子的 $D$ 和 $E$ 都不为零, 该疑惑迄今尚无满 意的理论解释. 本文根据该晶体的结构 ${ }^{[9]}$, 采用 $d^{9}-d^{9}$ 电子组态对模型: (i) 合理地解释了零场分 裂 $D, E$ 反常出现的疑惑; (ii) 用组态对内部的双跃迁机制解释了 $21000 \mathrm{~cm}^{-1}$ 谱带出现的原 因, 并预测 $25000 \mathrm{~cm}^{-1}$ 以上存在另一条双跃迁谱.

\section{1 理论处理}

根据晶场理论 ${ }^{[10]}$, 晶场中络离子中心金属离子哈密顿量为

$$
H=H_{f o}+V_{c f}+H_{s o} \text {, }
$$

式中第一项为自由离子哈密顿量; 第二项是晶场势; 第三项表示 $d$ 电子的自旋 - 轨道耦合.

当相邻络离子的两个中心金属离子间交换相互作用不能忽略时,式 (1)不再适用. 根据文献[8], 无外磁场和略去核能级贡献时, 哈密顿量为

$$
H=H_{1 f o}+H_{2 f o}+V_{1 c f}+V_{2 c f}+H_{1 s o}+H_{2 s o}+H_{s_{1} s_{2}},
$$

式中第一至六项的下标 1 和 2 分别对应中心金属离子 1 和 2 ; 最后一项 $H_{s_{1} s_{2}}=J S_{1} \cdot S_{2}$ 表示两 离子的交换相互作用, 计算中作微扰处理.

在低对称 $C_{2}$ 晶场中, 四配位络离子 (四面体) $d^{9}$ 电子组态中心金属离子的晶场势为

$$
V_{k c j}\left(C_{2}\right)=\sum_{j} V_{k}\left(j_{k}\right) \quad(k=1,2),
$$

式中 $V(j)$ 是单电子晶场势. 为简化, 略去表示离子 1 和 2 的足标 $k$ 后, $V(j)$ 可表为:

$$
\begin{aligned}
V(j)= & r_{j}^{2} \gamma_{20} Y_{2,0}\left(\theta_{j}, \varphi_{j}\right)+r_{j}^{2}\left(\gamma_{22}-i \gamma_{22}{ }^{\prime}\right) Y_{2,2}\left(\theta_{j}, \varphi_{j}\right)+r_{j}^{2}\left(\gamma_{22}+i \gamma_{22}{ }^{\prime}\right) Y_{2,-2}\left(\theta_{j}, \varphi_{j}\right)+ \\
& r_{j}^{4} \gamma_{40} Y_{4,0}\left(\theta_{j}, \varphi_{j}\right)+r_{j}^{4}\left(\gamma_{42}-i \gamma_{42}\right) Y_{4,2}\left(\theta_{j}, \varphi_{j}\right)+r_{j}^{4}\left(\gamma_{42}+i \gamma_{42}^{\prime}\right) Y_{4,-2}\left(\theta_{j}, \varphi_{j}\right)+ \\
& r_{j}^{4}\left(\gamma_{44}-i \gamma_{44}\right) Y_{4,4}\left(\theta_{j}, \varphi_{j}\right)+r_{j}{ }^{4}\left(\gamma_{44}+i \gamma_{44}{ }^{\prime}\right) Y_{4,-4}\left(\theta_{j}, \varphi_{j}\right),
\end{aligned}
$$

其中 $Y_{l, m}$ 是球谐函数 $(l=2,4 ; m=0, \pm 2, \pm 4)$; 参量

1994-12-21 收稿, 1995-03-11 收修改稿

*国家自然科学基金资助项目 


$$
\gamma_{l m}\left(\text { 和 } \gamma_{l m}{ }^{\prime}\right)=-\sum_{\tau} \frac{4 \pi e q \tau}{2 l+1} \frac{1}{R_{\tau}^{l+1}} Z_{l, m}\left(\Theta_{\tau}, \Phi_{\tau}\right),
$$

上两式中, $e$ 是中心金属离子的电子的电量; $\gamma_{j}, \theta_{j}, \varphi_{j}$ 是第 $j$ 个电子的坐标; $R_{\tau}, \Theta_{\tau}, \Phi_{\tau}$ 和 $q_{\tau}$ 分别是第 $\tau$ 个配位体的坐标和电量; $Z_{1, m}$ 是实球谐函数. 选取坐标使 $z \| c_{2}$ 轴, 根据 $c_{2}$ 对称结 构可计算出 $\gamma_{l m}\left(\right.$ 和 $\left.\gamma_{l m}{ }^{\prime}\right)$.

由 $c_{2}$ (双值群) 对称下 $d^{9}$ 电子组态的二重简并 (Kramers 简并) 基函数 $\left|\alpha_{n}\right\rangle$ 和 $\left|\beta_{n}\right\rangle$ 、哈密 顿量 (2) 式中的前六项以及晶场势 (4) 式和参量 (5) 式, 本文推出晶场、自旋 - 轨道耦合能量矩 阵 (见表 1).

表 $1 \quad c_{2}$ 对称下 $d^{9}$ 组态晶场及自旋 - 轨道耦合能量矩阵

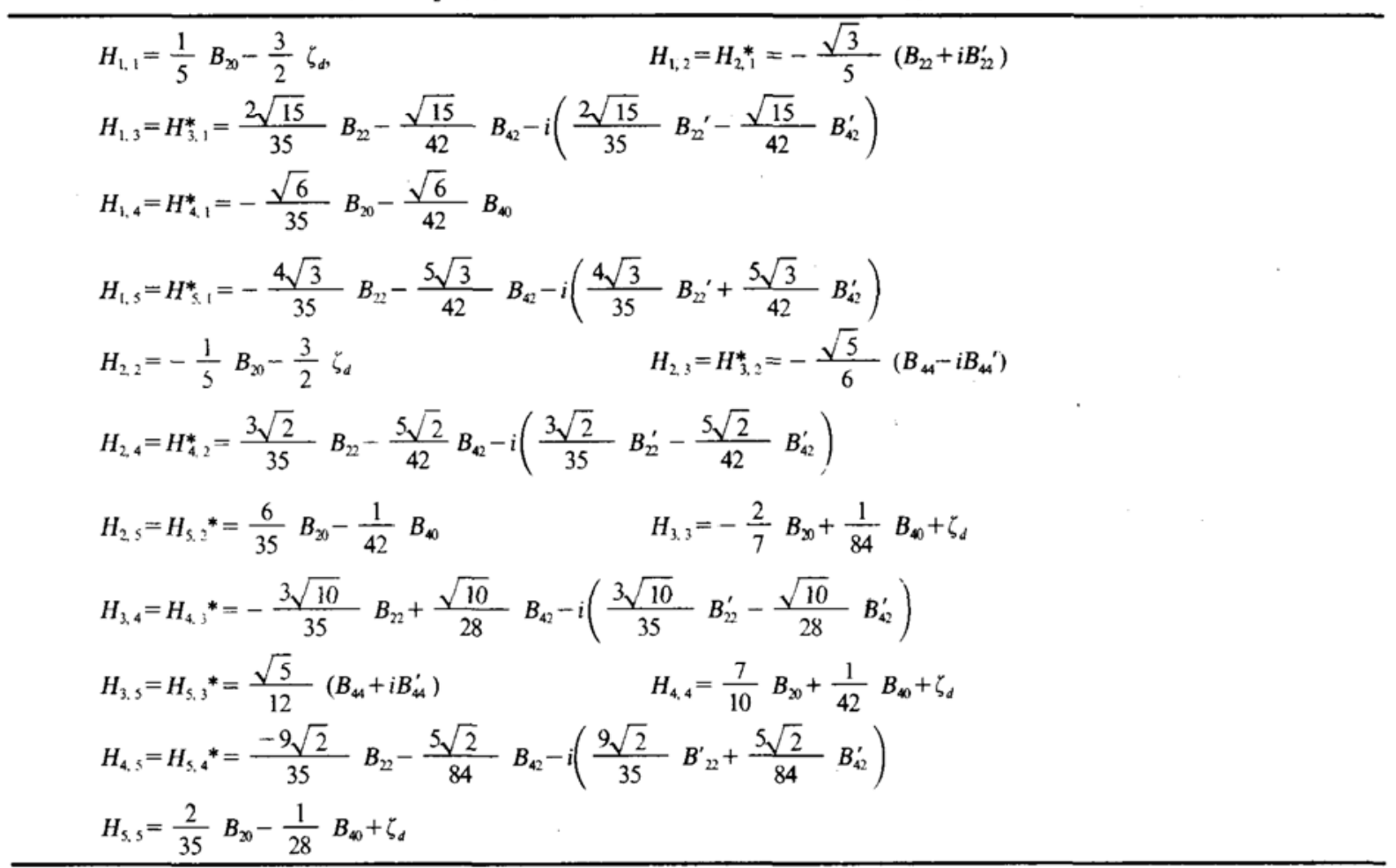

表 1 列出了两套简并能量矩阵 (Kramers 简并) 中的一套. $\zeta_{d}$ 是 $d$ 电子的自旋-轨道耦合常 数; 晶场参量 $B_{l m}\left(B_{l m}^{\prime}\right)$ 和参量 $\gamma_{l m}\left(\gamma_{l m}^{\prime}\right)$ 的联系为:

$$
\begin{aligned}
& B_{20}=\frac{1}{2} \sqrt{\frac{5}{\pi}}\left\langle r^{2}\right\rangle \gamma_{20}, B_{40}=\frac{6}{\sqrt{\pi}}\left\langle r^{4}\right\rangle \gamma_{40}, \quad B_{22}=\frac{1}{6} \sqrt{\frac{30}{\pi}}\left\langle r^{2}\right\rangle \gamma_{22}, \\
& B_{22}^{\prime}=\frac{1}{6} \sqrt{\frac{30}{\pi}}\left\langle r^{2}\right\rangle \gamma_{22}^{\prime}, B_{42}=\frac{3}{5} \sqrt{\frac{10}{\pi}}\left\langle r^{4}\right\rangle \gamma_{42}, B_{42}^{\prime}=\frac{3}{5} \sqrt{\frac{10}{\pi}}\left\langle r^{4}\right\rangle \gamma_{42}^{\prime}, \\
& B_{44}=\frac{6}{35} \sqrt{\frac{70}{\pi}}\left\langle r^{4}\right\rangle \gamma_{44}, B_{44}^{\prime}=\frac{6}{35} \sqrt{\frac{70}{\pi}}\left\langle r^{4}\right\rangle \gamma_{44}^{\prime} .
\end{aligned}
$$

采用完全对角化方法, 由表 1 的能量矩阵可解出 (i) 体系的能量和 (ii)二套简并的基态波函 数:

$$
|\Gamma(\alpha)\rangle=\sum_{n=1}^{5} a_{n}\left|\alpha_{n}\right\rangle, \quad|\Gamma(\beta)\rangle=\sum_{n=1}^{5} b_{n}\left|\beta_{n}\right\rangle,
$$


式中 $a_{n}, b_{n}$ 分别为两基态波函数的叠加系数.

当相邻络离子的两中心金属离子 $(M)$ 间交换相互作用不能忽略时, 将发生 $M-M$ 离子对 内部的双跃迁 ${ }^{[11]}$, 并对 EPR 零场分裂产生影响. 采用 $d^{9}-d^{9}$ 电子组态对模型时, 组态对总自旋

$$
S=S_{1}+S_{2} ; S_{1}+S_{2}-1 ; \cdots=1,0,
$$

耦合波函数为单离子波函数的线性叠加, 不同自旋分量的叠加波函数如下:

$$
\begin{aligned}
& \left|\Gamma\left(M_{s}=1\right)\right\rangle=\left|\Gamma_{1}(\alpha) \Gamma_{2}(\alpha)\right\rangle, \\
& \left|\Gamma\left(M_{s}=0\right)\right\rangle=\frac{1}{\sqrt{2}}\left[\left|\Gamma_{1}(\alpha) \Gamma_{2}(\beta)\right\rangle+\left|\Gamma_{1}(\beta) \Gamma_{2}(\alpha)\right\rangle\right], \\
& \left|\Gamma\left(M_{s}=-1\right)\right\rangle=\left|\Gamma_{1}(\beta) \Gamma_{2}(\beta)\right\rangle,
\end{aligned}
$$

式中下标 1 和 2 分别对应 $d^{9}$ 离子 1 和 2 .

考虑到体系内部磁相互作用, 各向异性 $g$ 因数可由下式求得,

$$
g_{i}=2\left\langle\Gamma\left(M_{S}=1\right)\left|L_{i}+g_{e} S_{i}\right| \Gamma\left(M_{S}=-1\right)\right\rangle, \quad(i=x, y, z),
$$

式中 $L_{i}$ 是轨道角动量分量算符, $S_{i}$ 是自旋角动量分量算符; $g_{e}=2.0023$ 是自由电子的 $g$ 因 数.

视 $d^{9}-d^{9}$ 组态对内部的交换相互作用 $J S_{1} \cdot S_{2}$ 为微扰, 由以下公式可分别求得零场分裂轴对 称分量 $D$ 和斜方分量 $E$ :

$$
\begin{gathered}
D=\left\langle\Gamma\left(M_{S}=1\right)\left|J S_{i} \cdot S_{2}\right| \Gamma\left(M_{S}=1\right)\right\rangle-\left\langle\Gamma\left(M_{S}=0\right)\left|J S_{1} \cdot S_{2}\right| \Gamma\left(M_{S}=0\right)\right\rangle, \\
E=\left\langle\Gamma\left(M_{S}=1\right)\left|J S_{1} \cdot S_{2}\right| \Gamma\left(M_{S}=-1\right)\right\rangle .
\end{gathered}
$$

以上低对称 $C_{2}$ 晶场 (四面体配位) 的公式适用面较广, 根据群论, 很容易由它们退化得到 适用于 $C_{2 v}, C_{2 h}, D_{2 d}, D_{2 h}$ 和 $T_{d}$ 对称晶场的公式.

\section{2 计算}

Schäfer 等 ${ }^{[9]}$ 的 $\mathrm{X}$ 射线衍射实验表明, $\left(\mathrm{C}_{5} \mathrm{H}_{5} \mathrm{NO}\right){ }_{2} \mathrm{CuCl}_{2}$ 具有单斜晶型结构, 空间对称为 $P 2_{1 / b}-C_{2 h}^{5}$, 络离子 $(2 \mathrm{Cl}-\mathrm{Cu}-20)^{4-}$ (四面体配位) 的局部点群对称近似为 $C_{2 v}$, 相邻络离子的两 个中心金属 $\mathrm{Cu}^{2+}$ 离子 $\left(d^{9}\right)$ 相距较近, 彼此共用两个 $\mathrm{O}^{2-}$ 离子配体 $\left(2 \mathrm{Cl}^{-}-\mathrm{Cu}^{2+}-2 \mathrm{O}^{2-}-\mathrm{Cu}^{2+}-\right.$ $\left.2 \mathrm{Cl}^{-}\right)$, 形成 $\mathrm{Cu}^{2+}-\mathrm{Cu}^{2+}\left(d^{9}-d^{9}\right)$ 离子对的链状结构, 离子对内部存在较强的交换相互作用 ${ }^{[8]}$. 在 $C_{2 V}$ 对称下, 上面公式中的晶场参量 $B_{22}^{\prime}, B_{42}^{\prime}$ 和 $B_{44}^{\prime}$ 为零. 本文采用晶场理论中流行的参量拟 合法, 根据 Kokoszka 等 ${ }^{[8]}$ 实验测得的 $\left(\mathrm{C}_{5} \mathrm{H}_{5} \mathrm{NO}\right)_{2} \mathrm{CuCl}_{2}$ 中 $\mathrm{Cu}^{2+}$ 离子的电子跃迁光谱数据, 拟 合得各晶场参量 $B_{l m}$ (见表 2). 将它们代人能量矩阵 (表 1) 并在计算机上求解, 得 (i) $d-d$ 跃迁 能级和 (ii) 形如 (7) 式的二重简并的基态波函数. 进一步在计算机上求解 (8) (11) 式, 得到各 向异性的 $g$ 因数和零场分裂 $D, E$ 值 (见表 2).

\section{3 结论}

(1) 本文采用 $d^{9}-d^{9}$ 组态对模型和考虑了组态对内部交换相互作用, 研究了 $\left(\mathrm{C}_{5} \mathrm{H}_{5} \mathrm{NO}\right)_{2}$ $\mathrm{CuCl}_{2}$ 的零场分裂, 使 $D$ 和 $E$ 不为零的疑惑得到合理的解释. 该方法适用于所有 $d^{9}-d^{9}\left(d^{1}-d^{1}\right)$ 离 子交换相互作用不能被忽略的情况. 当交换相互作用可忽略时退化为单个的 $d^{9}\left(d^{1}\right)$ 组态. 由 式 (10) 和 (11), 此时 $D$ 和 $E$ 为零.

(2) 电子跃迁光谱中, 关于 $21000 \mathrm{~cm}^{-1}$ 附近的谱带, Kokoszka ${ }^{[8]}$ 无法解释该谱产生的原 
表 2 理论计算和实验的比较

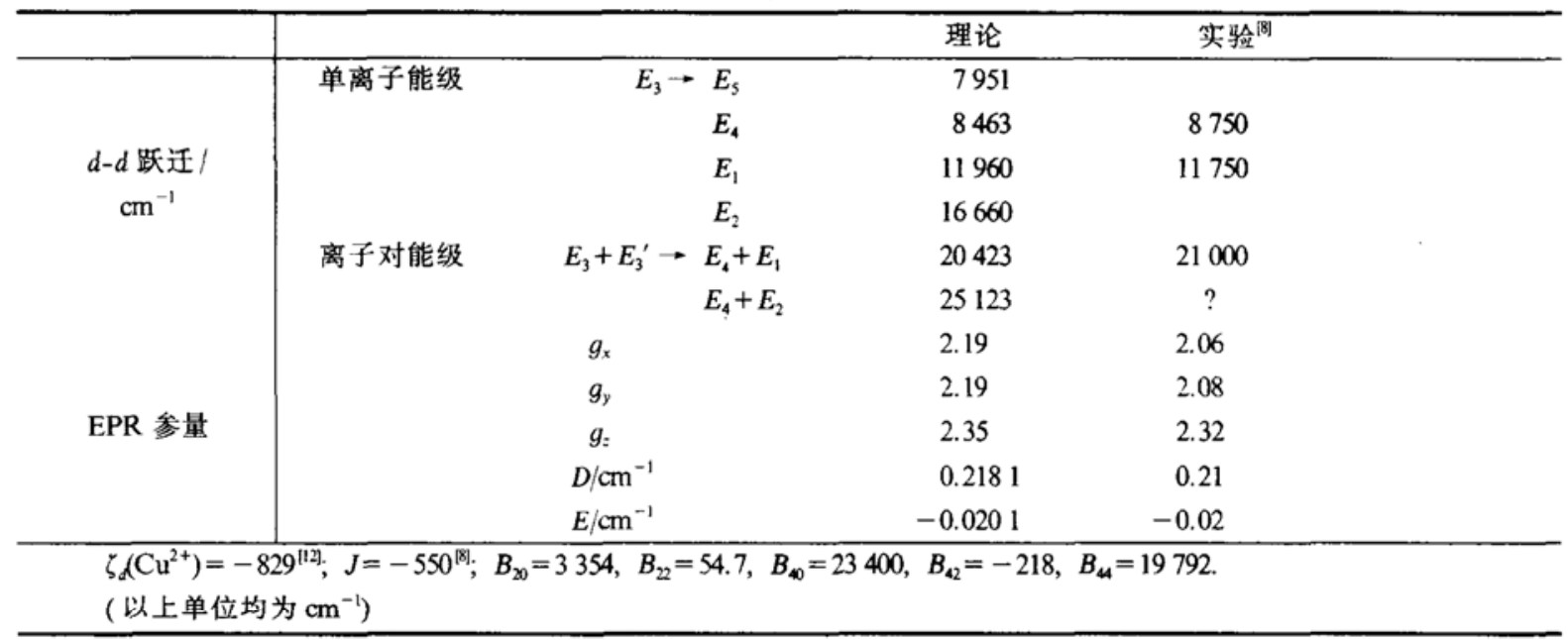

因, 本文用组态对内部的双跃迁计算出该谱带来自于 $E_{3}+E_{3}^{\prime} \rightarrow E_{4}+E_{1}$ 的贡献. 并且, 理论计 算还预测在 $25000 \mathrm{~cm}^{-1}$ 以上位置, 存在另一条双跃迁谱: $E_{3}+E_{3}{ }^{\prime} \rightarrow E_{4}+E_{2}$, 这一预测有待实 验证实.

\section{参 考 文 献}

1 Chand P. Upreti G C. Electron paramagnetic resonance study of $\mathrm{Cu}^{2+}$ - doped ammonium iodide single crystals. Chem Phys Letters, 1982, 88(3): 309 314

2 Arambuu J A, Moreno M. Bonding in $d^{9}$ complexes derived from EPR: application to $\mathrm{CuCl}_{4}^{2-}, \mathrm{CuBr}_{4}^{2-}$ and $\mathrm{CdCl}_{2}$ : $\mathrm{Cu}^{2+}$. J Chem Phys, 1985, 83(12):6071 6083

3 Misra S K, Wang C. EPR and optical-absorption studies of $\mathrm{Cu}^{2+}$ - doped $\mathrm{Mg}\left(\mathrm{CH}_{3} \mathrm{COO}\right)_{2} 4 \mathrm{H}_{2} \mathrm{O}$ single crystal. Phys Stat Sol(b), 1989, 154:259 271

4 Moreno M, Arambuu J A. An alternative interpretation of the "anomalous" positive g-shift observed for $\mathrm{NH}_{4} \mathbf{I}$ : $\mathrm{Cu}^{2+}$. Chem Phys Letters, 1984, 108(5):513 517

5 Kuwabara C. ESR investigation of $3 d^{9}$ ions alkali-halides. J Phys Soc Japan, 1971, 31(4): 1 074 1084

6 Boettcher F, Spaeth J M. ENDOR investigation of $\mathrm{Cu}^{++}$œntres in $\mathrm{NH}_{4} \mathrm{Cl}$. Phys Stat Sol(b), 1974, 61: 465 473

7 Zhou Y Y. A theoretical study of EPR spectra in $\mathrm{Cu}^{2+}$ and $\mathrm{Mn}^{2+}$ doped $\mathrm{CaCd}\left(\mathrm{CH}_{3} \mathrm{COO}\right)_{4} 6 \mathrm{H}_{2} \mathrm{O}$. Phys Stat Sol(b), 1988, 147: 273 280

8 Kokoszka G F, Allen H C Jr, Gordon G. Magnetic and optical spectra of two dimeric copper chloride pyridine-Noxide complexes. J Chem Phys. 1967, 46(8):3013 3019

9 Schäfer H I, Morrow J C, Smith H M. Magnetic susceptibility and crystal structure of (Pyridine N-oxide)-oopper (II) chloride. J Chem Phys, 1965, 42(2): $504 \sim 508$

$10 \mathrm{Yu} \mathrm{W} \mathrm{L,} \mathrm{Wang} \mathrm{J} \mathrm{Z.} \mathrm{Zero-field} \mathrm{splitting} \mathrm{and} \mathrm{the} \mathrm{optical} \mathrm{fine} \mathrm{structure} \mathrm{in} \mathrm{Fe}^{3+}: \mathrm{Al}_{2} \mathrm{O}_{3}$ crystals. Phys Stat Sol(b), 1993, 176: $433 \sim 439$

11 Siu G G, Zhao M G. Electron-spin-resonance studies of $\mathrm{Cr}$-ion pairs in $\mathrm{LiNbO}_{3} ; \mathrm{Cr}^{3+}$. Phys Rev, 1991, B43(16): $13575 \sim 13578$

12 Parker I H. $\mathrm{Cu}^{2+}$ in ammonium fluoride-a tetrahedral site. J Phys C:Solid St Phys, 1971, 4:2967 2978 